\title{
Numerical solution of compressible fluid flow in porous media with boundary element method
}

\author{
R. Jecl ${ }^{1}$, L. Škerget ${ }^{2}$ \& J. Kramer ${ }^{1}$ \\ ${ }^{I}$ Faculty of Civil Engineering, University of Maribor, Slovenija \\ ${ }^{2}$ Faculty of Mechanical Engineering, University of Maribor, Slovenija
}

\begin{abstract}
In the present work a fully developed boundary element method numerical scheme is presented for the simulation of compressible fluid flow in porous media with restriction to the subsonic flows. The flow is modeled by utilizing the Brinkman extended Darcy momentum equation which is commonly used when it is important to satisfy the non-slip boundary condition on impermeable surfaces that bound the porous media domain. The governing equations are transformed by using the velocity-vorticity variables formulation and therefore the computation scheme is partitioned into kinematic and kinetic part. The method is applied to consider buoyancy driven flow in closed porous cavity, differentially heated under large temperature gradients. The results in terms of velocity and temperature redistribution as well as the total heat transfer across the cavity will be presented for different governing parameters.

Keywords: porous media, compressible fluid flow, boundary domain integral method, boundary element method, natural convection.
\end{abstract}

\section{Introduction}

Most of the studies dealing with transport phenomena in porous media are based on presuming the fluid is incompressible and viscous, where the mass density is a constant quantity the velocity does not depend on the mass density and pressure is simply a force in the linear momentum balance equation. In this work, the boundary element method, which has been established for the viscous incompressible fluid motion [1], is modified and extended to capture the compressible fluid state with restriction to the subsonic flows. That means that 
the difference in mass density significantly changes the velocity field but there are no shock waves and no sudden sharp changes in the values of the field functions. Furthermore the pressure is a thermodynamic quantity which is temperature and mass density dependent.

\section{Mathematical formulation}

The present work is concerned with natural convection in a two-dimensional, rectangular enclosure with the vertical walls held at different temperatures and the connecting horizontal walls considered adiabatic. The enclosure is filled with porous material, which is homogeneous and isotropic, and the fluid that saturates the solid matrix and both are in local thermodynamic equilibrium. The flow is assumed to be steady, laminar, and compressible. The general set of equations for conservation of mass, momentum and energy are based on the Brinkman extended Darcy flow model. The equations governing the conservation of mass, momentum and energy can be written as follows [2]

$$
\begin{gathered}
\frac{\partial \phi \rho}{\partial t}+\frac{\partial \rho v_{j}}{\partial x_{j}}=0 \\
\rho\left[\frac{\partial v_{i}}{\partial t}+v_{j} \frac{\partial}{\partial x_{j}}\left(\frac{v_{i}}{\phi}\right)\right]=-\phi \frac{\partial p}{\partial x_{j}}+\phi \rho g_{i}-\mu \frac{\partial^{2} v_{i}}{\partial x_{j} \partial x_{j}} \\
+(\mu+\hat{\mu}) \frac{\partial^{2} v_{j}}{\partial x_{i} \partial x_{j}}-\frac{\mu \phi}{K} v_{j}, \\
\frac{\partial}{\partial t}\left[\phi\left(\rho c_{p}\right)_{f}+(1-\phi)\left(\rho c_{p}\right)_{s}\right] T+\frac{\partial}{\partial x_{j}}\left[\phi\left(\rho c_{p}\right)_{f} \frac{v_{j}}{\phi} T\right]=\frac{\partial}{\partial x_{j}} \lambda_{e} \frac{\partial T}{\partial x_{j}}
\end{gathered}
$$

The parameters, used above are: $v_{i}$ volume-averaged velocity, $x_{i}$ the i-th coordinate, $\phi$ porosity, $t$ time, $\rho$ density, $\mu$ dinamic viscosity, $\partial p / \partial x_{j}$ the pressure gradient, $g_{i}$ gravity, $K$ permeability of porous media, $\hat{\mu}$ coefficient of bulk viscosity, $T$ is temperature, $\lambda_{e}$ the effective thermal conductivity of the porous media given as $\lambda_{e}=\phi \lambda_{f}+(1-\phi) \lambda_{s}$, where $\lambda_{f}$ and $\lambda_{s}$ are thermal conductivities for the fluid and solid phases, respectively. Furthermore $\left(\rho c_{p}\right)_{f}$ and $\left(\rho c_{p}\right)_{s}$ represent heat capacity for the fluid and solid phases. Introducing new variables $\rho^{\prime}=\rho \phi$ and $v_{j}^{\prime}=v_{j} / \phi$, and with the definition of Stokes material derivative of the variable (.) as $D(\cdot) / D t=\partial(\cdot) / \partial t+v_{k} \partial(\cdot) / \partial x_{k}$, continuity equation can be written as 


$$
\frac{\partial v_{j}^{\prime}}{\partial x_{j}}=D^{\prime}=-\frac{1}{\rho^{\prime}} \frac{D \rho^{\prime}}{D t},
$$

where $D^{\prime}$ represent a local expansion rate. According to Stokes hypothesis the second viscous coefficient can be taken as $\hat{\mu}=-2 / 3 \mu$. Because of analytical reasons to develop velocity-vorticity formulation of governing equations, the momentum eq. (2) is worth writing in its extended form [3, 4]

$$
\begin{gathered}
\rho \frac{D v_{i}^{\prime}}{D t}=-e_{i j k} \frac{\partial \mu \omega_{k}^{\prime}}{\partial x_{j}}+2 e_{i j k} \frac{\partial \mu}{\partial x_{j}} \omega_{k}^{\prime}+2 \frac{\partial \mu}{\partial x_{j}} \frac{\partial v_{i}^{\prime}}{\partial x_{j}}-\frac{2}{3} \frac{\partial \mu}{\partial x_{i}} D^{\prime} \\
+\frac{4}{3} \mu \frac{\partial D^{\prime}}{\partial x_{i}}-\frac{\partial p}{\partial x_{j}}+\rho g_{i}-\frac{\mu \phi}{K} v_{j}^{\prime}
\end{gathered}
$$

with the introduction of the vorticity vector $\omega$, representing the curl of the velocity vector,

$$
\omega_{i}=e_{i j k} \frac{\partial v_{k}}{\partial x_{j}}, \quad \frac{\partial \omega_{j}}{\partial x_{j}}=0,
$$

and having in mind that in our case the original vorticity is replaced by the socalled compressible vorticity $\omega^{\prime}, \omega^{\prime}=(1 / \phi) \omega$. It is important to stress out that porosity $\phi$ is taken to be constant over individual subdomain but changeable in respect to the whole computational domain.

Representing the material properties as a sum of a constant and variable part

$$
\mu=\mu_{0}+\tilde{\mu}, \quad \rho=\rho_{0}+\tilde{\rho}, \quad\left(\rho^{\prime} c_{p}\right)_{f}=c_{0}+\tilde{c}, \quad \lambda_{e}=\lambda_{e 0}+\tilde{\lambda},
$$

then the momentum and energy equations (3) and (5) can be written as

$$
\begin{gathered}
\frac{D v_{i}^{\prime}}{D t}=-e_{i j k} \frac{\partial \mu_{0} \omega_{k}^{\prime}}{\partial x_{j}}-\frac{1}{\rho_{0}} \frac{\partial p}{\partial x_{j}}+\frac{\rho}{\rho_{0}} g_{i}+\frac{1}{\rho_{0}} f_{i}^{m}-\frac{\mu \phi}{\rho_{0} K} v_{j}^{\prime}, \\
\frac{D T}{D t}=a \frac{\partial^{2} T}{\partial x_{j} \partial x_{j}}+\frac{S_{T}^{m}}{c_{0}},
\end{gathered}
$$

where $a$ is thermal diffusivity, and the pseudo body force $f_{i}^{m}$ and pseudo heat source $S_{T}^{m}$ terms include the effects of variable material properties, given by

$$
f_{i}^{m}=-e_{i j k} \frac{\partial \tilde{\mu} \omega_{k}^{\prime}}{\partial x_{j}}+2 e_{i j k} \frac{\partial \mu}{\partial x_{j}} \omega_{k}^{\prime}+2 \frac{\partial \mu}{\partial x_{j}} \frac{\partial v_{i}^{\prime}}{\partial x_{j}}-\frac{2}{3} \frac{\partial \mu}{\partial x_{i}} D^{\prime}+\frac{4}{3} \mu \frac{\partial D^{\prime}}{\partial x_{i}}-\tilde{\rho} a_{i}
$$




$$
S_{T}^{m}=\frac{1}{c_{0}}\left(-\frac{\partial}{\partial x_{j}} \tilde{\lambda}_{e} \frac{\partial T}{\partial x_{j}}-T \rho^{\prime} \frac{D\left(c_{p}\right)_{f}}{D t}+\frac{\partial\left[(1-\phi)\left(\rho c_{p}\right)_{s}\right] T}{\partial t}+\widetilde{c} \frac{D T}{D t}\right) .
$$

\section{Numerical method}

The numerical method chosen for this investigation is the Boundary Domain Integral Method based on the classical Boundary Element Method.

The kinematic is given by velocity vector Poisson's equation

$$
\frac{\partial^{2} v_{i}^{\prime}}{\partial x_{j} \partial x_{j}}+e_{i j k} \frac{\partial \omega_{k}^{\prime}}{\partial x_{j}}-\frac{\partial D^{\prime}}{\partial x_{i}}=0
$$

representing the mass conservation equation (1) and the vorticity definition (6), expressing the compatibility and restriction conditions between velocity, vorticity, and mass density field functions. To accelerate the convergence and the stability of the coupled velocity, vorticity and pressure computational iterative scheme, the false transient approach may be used for equation (12) [5]. By adding the artificial accumulation term it can be written in its parabolic diffusion form

$$
\frac{\partial^{2} v_{i}^{\prime}}{\partial x_{j} \partial x_{j}}-\frac{1}{\alpha} \frac{\partial v_{i}^{\prime}}{\partial t}+e_{i j k} \frac{\partial \omega_{k}^{\prime}}{\partial x_{j}}-\frac{\partial D^{\prime}}{\partial x_{i}}=0
$$

where $\alpha$ is the relaxation parameter controlling the diffusion and accumulation processes. It is obvious that the governing velocity equation (12) is exactly satisfied only at the steady state of the artificial transient $(t \rightarrow \infty)$, when the false time derivative vanishes. For the two-dimensional plane flow the vorticity vector has just one component perpendicular to the plane of the flow, e.g. $\omega_{k}^{\prime}=\left(0,0, \omega^{\prime}\right)$, therefore the equation (13) reduces to

$$
\frac{\partial^{2} v_{i}^{\prime}}{\partial x_{j} \partial x_{j}}-\frac{1}{\alpha} \frac{\partial v_{i}^{\prime}}{\partial t}+e_{i j} \frac{\partial \omega}{\partial x_{j}}-\frac{\partial D^{\prime}}{\partial x_{i}}=0
$$

The vorticity kinetics is given by the vorticity transport equation obtained as a curl of the momentum equation (8) in the form

$$
\begin{gathered}
\frac{D \omega_{i}^{\prime}}{D t}=v_{0} \frac{\partial^{2} \omega_{i}^{\prime}}{\partial x_{j} \partial x_{j}}+\omega_{j}^{\prime} \frac{\partial v_{i}^{\prime}}{\partial x_{j}}-\omega_{i}^{\prime} D^{\prime}+\frac{1}{\rho_{0}} e_{i j k} \frac{\partial \rho g_{k}}{\partial x_{j}} \\
+\frac{1}{\rho_{0}} e_{i j k} \frac{\partial f_{i}^{m}}{\partial x_{j}}-\frac{\mu \phi}{\rho_{0} K} \omega_{i}^{\prime} .
\end{gathered}
$$


The vorticity transport equation (15) is a highly nonlinear partial differential equation due to the products of velocity and vorticity having in mind that the velocity is kinematically dependent on vorticity. For the two-dimensional plane flow equation (15) is reduced to a scalar equation for vorticity $\omega^{\prime}$

$$
\frac{D \omega^{\prime}}{D t}=v_{0} \frac{\partial^{2} \omega_{i}^{\prime}}{\partial x_{j} \partial x_{j}}-\frac{1}{\rho_{0}} e_{i j} \frac{\partial \rho g_{i}}{\partial x_{j}}-\frac{1}{\rho_{0}} e_{i j} \frac{\partial f_{i}^{m}}{\partial x_{j}}-\frac{\mu \phi}{\rho_{0} K} \omega,
$$

reducing also the pseudo body force term $f_{i}^{m}$ to

$$
f_{i}^{m}=-e_{i j} \frac{\partial \tilde{\mu} \omega^{\prime}}{\partial x_{j}}+2 e_{i j} \frac{\partial \mu}{\partial x_{j}} \omega^{\prime}+2 \frac{\partial \mu}{\partial x_{j}} \frac{\partial v_{i}^{\prime}}{\partial x_{j}}-\frac{2}{3} \frac{\partial \mu}{\partial x_{i}} D^{\prime}+\frac{4}{3} \mu \frac{\partial D^{\prime}}{\partial x_{i}}-\tilde{\rho} a_{i} .
$$

Due to the buoyancy force and variable material property terms, acting as additional temperature and pressure dependent vorticity source terms, the vorticity transport equation is coupled to the energy and pressure equations, making the numerical procedure very severe.

In the compressible fluid dynamics the pressure is a thermodynamic quantity which is temperature and mass density dependent. Writing the momentum equation (8) for the pressure gradient we have

$$
\frac{\partial p}{\partial x_{i}}=f_{p i}=-\mu_{0} e_{i j} \frac{\partial \omega^{\prime}}{\partial x_{j}}-\rho_{0} a_{i}+\rho g_{i}+f_{i}^{m}-\frac{\mu \phi}{K} v_{j}^{\prime} .
$$

To derive the pressure equation, depending on known field and material functions, the divergence of equation (18) should be calculated, resulting in the elliptic Poisson pressure equation

$$
\frac{\partial^{2} p}{\partial x_{i} \partial x_{i}}-\frac{\partial f_{p i}}{\partial x_{i}}=0
$$

As above with velocity vector equation, the false pressure transient term may be added to obtain false parabolic diffusion equation

$$
\frac{\partial^{2} p}{\partial x_{i} \partial x_{i}}-\frac{1}{\alpha} \frac{\partial p}{\partial t}-\frac{\partial f_{p i}}{\partial x_{i}}=0
$$

Equations (9), (14), (16) and (20) represent the leading non-linear set of equations to which the weighted residuals technique of the BDIM has to be applied [6]. Each of these equations can be written as in the following general differential conservation equation 
148 Fluid Structure Interaction and Moving Boundary Problems IV

$$
\aleph[u]+b=0,
$$

where the linear differential operator $\aleph[u]$ will be parabolic diffusion differential operator of the form

$$
\aleph[u]=a_{i} \frac{\partial^{2} u}{\partial x_{j} \partial x_{j}}-\frac{\partial u}{\partial t},
$$

with $a_{i}$ equal to: $\alpha$ for kinematics and pressure, $v_{0}$ and $a_{0}=\lambda_{e 0} / c_{0}$ for vorticity and temperature kinetics, respectively.

As the computational results of the present work are limited to the twodimensional case, all the subsequent integral equations will consequently be written for the case of planar geometry only. Integral representation of the kinematic equation is given with the following equation

$$
\begin{gathered}
c(\xi) v_{i}^{\prime}\left(\xi, t_{F}\right)+\alpha \int_{\Gamma} \int_{t_{F-1}}^{t_{F}} v^{\prime} \frac{\partial u^{*}}{\partial n} d t d \Gamma=\alpha \int_{\Gamma} \int_{t_{F-1}}^{t_{F}} \frac{\partial v_{i}^{\prime}}{\partial n} u^{*} d t d \Gamma \\
+\int_{\Omega} \int_{t_{F-1}}^{t_{F}} b u^{*} d t d \Omega+\int_{\Omega} v_{i, F-1}^{\prime} u_{F-1}^{*} d \Omega,
\end{gathered}
$$

where

$$
b=\alpha\left(e_{i j k} \frac{\partial \omega_{k}^{\prime}}{\partial x_{j}}-\frac{\partial D^{\prime}}{\partial x_{i}}\right) .
$$

The vorticity transport equation is given with the integral representation

$$
\begin{gathered}
c(\xi) \omega^{\prime}\left(\xi, t_{F}\right)+v_{0} \int_{\Gamma} \int_{t_{F-1}}^{t_{F}} \omega^{\prime} \frac{\partial u^{*}}{\partial n} d t d \Gamma=v_{0} \int_{\Gamma}^{t_{t_{F-1}}} \frac{\partial \omega^{\prime}}{\partial n} u^{*} d t d \Gamma \\
+\int_{\Omega} \int_{t_{F-1}}^{t_{F}} b u^{*} d t \quad d \Omega+\int_{\Omega} \omega_{i, F-1}^{\prime} u_{F-1}^{*} d \Omega,
\end{gathered}
$$

where

$$
b=-\frac{1}{\rho} \frac{\partial}{\partial x_{j}}\left(\rho_{0} v_{j}^{\prime} \omega^{\prime}+e_{i j} \rho g_{i}+e_{i j} f_{i}^{m}\right)-\frac{\mu \phi}{\rho_{0} K} \omega^{\prime} .
$$

Applying similar procedure to the heat transport equation (9), we have

$$
\begin{gathered}
c(\xi) T\left(\xi, t_{F}\right)+a \int_{\Gamma} \int_{t_{F-1}}^{t_{F}} T \frac{\partial u^{*}}{\partial n} d t d \Gamma=a \int_{\Gamma} \int_{t_{F-1}}^{t_{F}} \frac{\partial T}{\partial n} u^{*} d t d \Gamma \\
+\int_{\Omega}^{t_{t_{F-1}}} b u^{*} d t d \Omega+\int_{\Omega} T_{i, F-1} u_{F-1}^{*} d \Omega,
\end{gathered}
$$


with

$$
\begin{aligned}
& b=-\frac{1}{c_{0}} \frac{\partial}{\partial x_{j}}\left(\tilde{\lambda}_{e} \frac{\partial T}{\partial x_{j}}-c v_{j}^{\prime} T\right)-\frac{1}{c_{0}}\left(T v_{j}^{\prime} \frac{\partial c}{\partial x_{j}}+c T D^{\prime}+\widetilde{c} \frac{\partial T}{\partial t}\right) \\
& -\frac{1}{c_{0}} \frac{\partial\left[(1-\phi)\left(\rho c_{p}\right)_{s}\right] T}{\partial t}+\frac{1}{c_{0}} T \rho^{\prime} \frac{D\left(c_{p}\right)_{f}}{D t} .
\end{aligned}
$$

Finally, the pressure equation may be rewritten in the following integral statement

$$
\begin{gathered}
c(\xi) p\left(\xi, t_{F}\right)+\alpha \int_{\Gamma} \int_{t_{F-1}}^{t_{F}} p \frac{\partial u^{*}}{\partial n} d t d \Gamma=\alpha \int_{\Gamma} \int_{t_{F-1}}^{t_{F}} \frac{\partial p}{\partial n} u^{*} d t d \Gamma \\
+\int_{\Omega} \int_{t_{F-1}}^{t_{F}} b u^{*} d t d \Omega+\int_{\Omega} p_{F-1} u_{F-1}^{*} d \Omega,
\end{gathered}
$$

with

$$
b=\alpha \frac{\partial f_{p i}}{\partial x_{i}} .
$$

In all integral equations the constant variation of field functions $\left(v_{i}, \omega, T, p\right)$ is assumed within the individual time increment $\Delta t=t_{F}-t_{F-1}$, therefore the time integrals may be evaluated analytically,

$$
U^{*}=a_{i} \int_{t_{F-1}}^{t_{F}} u^{*} d t, Q^{*}=a_{i} \int_{t_{F-1}}^{t_{F}} \frac{\partial u^{*}}{\partial n} d t, Q_{i}^{*}=a_{i} \int_{t_{F-1}}^{t_{F}} \frac{\partial u^{*}}{\partial x_{i}} u^{*} d t
$$

Also in all equations $u^{*}$ is the parabolic diffusion fundamental solution in the form

$$
u^{*}=\frac{1}{4 \pi a_{i} \tau} e^{r^{2} / 4 a \tau},
$$

where the proper use of $a_{i}$ is as expressed above and $r$ is the magnitude of the vector from the source point to reference point. Final integral representation for velocity, vorticity, temperature and pressure are therefore given by the following equations

$$
\begin{gathered}
c(\xi) v_{i}^{\prime}\left(\xi, t_{F}\right)+\int_{\Gamma} v^{\prime} Q^{*} d \Gamma=\int_{\Gamma}\left(\frac{\partial v_{i}^{\prime}}{\partial n}-D^{\prime} n_{i}\right) U^{*} d \Gamma+e_{i j} \int_{\Gamma} \omega^{\prime} n_{j} U^{*} d \Gamma \\
-e_{i j} \omega_{\Omega} \omega^{\prime} Q_{j}^{*} d \Omega+\int_{\Omega} D^{\prime} Q_{i}^{*} d \Omega+\int_{\Omega} v_{i, F-1}^{\prime} u_{F-1}^{*} d \Omega,
\end{gathered}
$$




$$
\begin{gathered}
c(\xi) \omega^{\prime}\left(\xi, t_{F}\right)+\int_{\Gamma} \omega^{\prime} Q^{*} d \Gamma=\frac{1}{\mu_{0}} \int_{\Gamma}\left(\mu_{0} \frac{\partial \omega^{\prime}}{\partial n}-\rho_{0} v_{n}^{\prime} \omega^{\prime}+\rho g_{t}+f_{t}^{m}\right) U^{*} d \Gamma \\
+\frac{1}{\mu_{0}} \int_{\Omega}\left(\rho_{0} v_{j}^{\prime} \omega^{\prime}+\rho e_{i j} g_{i}+e_{i j} f_{i}^{m}\right) Q_{j}^{*} d \Omega \\
-\frac{1}{\mu_{0}} \int_{\Omega} \frac{\mu \phi}{K} \omega^{\prime} U^{*} d \Omega+\int_{\Omega} \omega_{i, F-1}^{\prime} u_{F-1}^{*} d \Omega, \\
c(\xi) T\left(\xi, t_{F}\right)+\int_{\Gamma} T Q^{*} d \Gamma=\frac{1}{\lambda_{e 0}} \int_{\Gamma} \lambda_{e 0} \frac{\partial T}{\partial n} U^{*} d \Gamma-\frac{1}{\lambda_{e 0}} \int_{\Omega} \tilde{\lambda}_{e} \frac{\partial T}{\partial x_{j}} Q_{j}^{*} d \Omega \\
+\frac{1}{\lambda_{e 0}} \int_{\Omega}\left(\begin{array}{c}
\left.T v_{j}^{\prime} \frac{\partial c}{\partial x_{j}}+c T D^{\prime}-\tilde{c} \frac{\partial T}{\partial t}-\frac{\partial\left[(1-\phi)\left(\rho c_{p}\right)_{s}\right] T}{\partial t}-T \rho^{\prime} \frac{D\left(c_{p}\right)_{f}}{D t}\right) \\
+\int_{\Omega} T_{i, F-1} u_{F-1}^{*} d \Omega, \\
c(\xi) p\left(\xi, t_{F}\right)+\int_{\Gamma} p Q^{*} d \Gamma=\int_{\Omega} f_{p i} Q_{i}^{*} d \Omega+\int_{\Omega} p_{F-1} u_{F-1}^{*} d \Omega .
\end{array}\right.
\end{gathered}
$$

where $v_{n}^{\prime}, g_{t}$ and $f_{t}^{m}$ are the normal velocity, tangential gravity and nonlinear material source components, respectively.

For the numerical approximate solution of the field functions, the integral equations are further written in a discretized manner in which the integrals over the boundary $\Gamma$ and domain $\Omega$ are approximated by a sum of the integrals over all boundary elements and over all internal cells. In such a way we obtain the matrix form of the equations, which are solved by coupling kinetic and kinematic equations, considering the corresponding boundary and initial conditions. Since the implicit set of equations is written simultaneously for all boundary and internal nodes, these procedure results in a very large fully populated system matrix influenced by diffusion and convection. The consequence of this approach is a very stable and accurate numerical scheme with substantial computer time and memory demands. To improve the economics of the computation, the subdomain technique is used, where the entire solution domain is partitioned into subdomains to which the same described numerical procedure can be applied [6]. The final system of equations for the entire domain is then obtained by adding the sets of equations for each subdomain considering the compatibility and equilibrium conditions between their interfaces, resulting in a much sparse system matrix suitable to solve with iterative techniques.

\section{Conclusion}

The boundary element integral approach to the solution of compressible fluid motion in thermally driven porous cavity is presented. The derived numerical 
model is characterized by the decomposition of flow into its kinematics and kinetics, a result of the velocity-vorticity formulation of the modified (porous) Navier-Stokes equation for a compressible fluid. The described numerical algorithm leads to strong coupling between velocity, vorticity and mass density fields, resulting in a stable numerical scheme. The application of the parabolic diffusion fundamental solution in the derivation of final integral representations ensures an accurate computation of the flow field variables. A numerical model based on the presented theoretical work is, at this moment, in the phase of evaluation and testing, therefore the results of test examples should be presented at the conference.

\section{References}

[1] Jecl, R., Škerget, L., Boundary element method for natural convection in non-Newtonian fluid saturated square porous cavity, Eng. Anal. Bound. Elem., 27, pp. 963-975, 2003.

[2] Bear, J., Bachmat, Y., Introduction to Modeling of Transport Phenomena in Porous Media, Kluwer Acad. Publ., Dordrecht, Boston \& London, 1991.

[3] Škerget, L., Jecl, R., Compressible fluid dynamics in porous media by the Boundary Element Method (Chapter 6). Emerging Technologies and Techniques in Porous Media, eds.: D.B. Ingham, A. Bejan, E. Mamut, I. Pop, Kluwer Acad. Publ., Dordrecht, Boston \& London, 93-117, 2004.

[4] Škerget, L., Samec, N., BEM for the two-dimensional plane compressible fluid dynamics, Eng. Anal. Bound. Elem., 29, pp. 1-17, 2005.

[5] Škerget, L., Rek, Z., Boundary-domain integral method using a velocityvorticity formulation, Eng. Anal. Bound. Elem., 15, pp. 359-370, 1995.

[6] Hriberšek, M., Škerget, L., Iterative methods in solving Navier-Stokes equations by the boundary element method, Int. J. Numer. Meth. Engng., 39, pp. 115-139, 1996. 